\title{
Relationship between the Levels of MMP-9, TIMP-1, and Zinc in Biological Samples of Patients with Carotid Atherosclerosis
}

\author{
Zakhro A. Usmanova \\ Tashkent Institute of Postgraduate Medical Education \\ Tashkent, Uzbekistan
}

\begin{abstract}
The aim our study was to evaluate the levels of zinc in blood serum, hair, and specimens of carotid artery atherosclerotic plaques (CAAPs) and their relationship to levels of MMP-9 and TIMP-1 in the serum of patients with stable and unstable CAAPs.

Material and Methods: The study included 73 patients (55 men and 18 women) aged from 46 to 88 years (mean age $65.96 \pm 1.07$ years) with CAAPs. The control group consisted of 10 healthy subjects of similar age and gender. The patients were divided into two groups depending on their atherosclerotic plaque stability according to prior duplex ultrasonography. Group 1 consisted of 45 patients with stable atherosclerotic plaque, and Group 2 included 28 patients with unstable AP. Patients with hemodynamically significant carotid stenosis and unstable atherosclerotic plaques underwent carotid endarterectomy. The serum concentration of MMP-9 and TIMP-1 was determined using the standard test systems for immunoassay. Quantitative determination of the zinc level in hair and atherosclerotic plaque was carried out by optical emission spectrometry; the serum Zn was determined colorimetrically.

Results: The serum levels of MMP-9 and TIMP-1 were significantly higher in Group 2 compared to Group1 and the control group. The index of MMP-9/TIMP-1 was 1.6 times higher in Group 2 compared to the control group. The level of $\mathrm{Zn}$ in serum and hair was not significantly different between Groups 1 and 2. However, Zn levels in unstable atherosclerotic plaque were lower than in the control group. Reducing the concentration of zinc in the hair was accompanied by a decrease in zinc level in atherosclerotic plaque specimens. With the progression of atherosclerosis and increasing the intima-media thickness of the common carotid artery, the level of zinc in serum and atherosclerotic plaques decreased. Increasing the serum concentration of MMP-9 was accompanied by decreasing the zinc level in atherosclerotic plaque. High serum concentration of MMP-9 and TIMP-1, and MMP-9/TIMP-1 imbalance testify to CAAP instability.
\end{abstract}

Keywords: carotid atherosclerosis; atherosclerotic plaque; matrix metalloproteinase-9 (MMP-9); TIMP metallopeptidase inhibitor 1; zinc.

\section{Introduction}

The instability of atherosclerotic plaque (AP) is the main cause of myocardial infarction (MI) and stroke [1]. Carotid artery AP (CAAP) proneness to rupture is an independent risk factor of acute coronary syndrome and MI [2]. The vulnerability of the atherosclerotic plaques depends on many factors, including endothelial function, presence of inflammatory cells, cytokine production, smooth muscle cells contents, and cell death (including necrosis and apoptosis) [3]. Vulnerable APs have a thin fibrous cap, a large lipid-rich

*Corresponding author: Zakhro A. Usmanova. Tashkent Institute of Postgraduate Medical Education; Tashkent, Uzbekistan E-mail: zahro.usmanova@yandex.ru necrotic core, and increased plaque inflammation with the elevated expression of matrix metalloproteinases (MMPs).

The activity of MMPs in various tissues is tightly regulated by endogenous tissue inhibitors of MMPs (TIMPs). Alteration of the fine physiological balance between MMPs and TIMPs may contribute to the pathophysiology of atherosclerosis [4]. MMPs are zinc-containing enzymes that are implicated in degradation and remodeling of components of the extracellular matrix. Increased levels of MMPs, and in particular of MMP-9 (gelatinase B), have been associated with several pathological inflammatory conditions, vascular diseases, and the instability of carotid atherosclerosis [58]. The catalytic domain of all MMPs contains a Zn2+ ion coordinated by a tris(histidine) motif; the $\mathrm{Zn} 2+$ ion is critical for both substrate binding and cleavage [9-11]. There is evidence that zinc blocks calcium and its several actions on 
atherogenesis. Increased amounts of cytotoxic cytokines, such as TNF-alpha, IL-beta and IL-8, often produced in the elderly, are blocked by high-dose zinc [12]. It has been established that there is a relationship between zinc deficiency in different biosubstrates and the development of obesity, type 2 diabetes mellitus, hypertension, coronary heart disease (CHD), and atherosclerosis.

We aimed to study the levels of zinc in blood serum, hair, and specimens of CAAPs and their relationship to levels of MMP-9 and TIMP-1 in the serum of patients with stable and unstable CAAPs.

\section{Material and Methods}

The study was approved by the Tashkent Institute of Postgraduate Medical Education Ethics Committee. The study included 73 patients (55 men and 18 women) aged from 46 to 88 years (mean age $65.96 \pm 1.07$ years) with CAAPs. The control group consisted of 10 healthy subjects of similar age and gender. Written informed consent was obtained from each patient. All patients were examined by a neurologist, cardiologist and vascular surgeon. The patients were divided into two groups depending on their AP stability according to prior duplex ultrasonography. Group 1 consisted of 45 patients with stable AP, and Group 2 included 28 patients with unstable AP.

Exclusion criteria were acute myocardial infarction, cardiomyopathy, acute myocarditis, pericarditis, acute ischemic stroke, malignant tumors, diffuse connective tissue diseases, acute infectious disease, pulmonary fibrosis and severe chronic obstructive pulmonary disease, left ventricular ejection fraction less than $45 \%$.

\section{Carotid duplex ultrasonography (CUS)}

All participants underwent a color duplex sonography of the entire extracranial carotid system using a duplex scanner HD3 (Phillips, The Netherlands) and a linear transducer (5.0$10.0 \mathrm{MHz}$ ). The calculation of the degree of stenosis (DS) was performed in the zone of maximum narrowing of the artery lumen. We investigated the common carotid artery (CCA), internal carotid artery (ICA), and carotid bifurcations for the presence of AP. The intima-media thickness of CCA (IMTCCA) was measured both at a standard point $(1 \mathrm{~cm}$ before the carotid bifurcation) and in AP (maximum IMT). IMT-CCA $>1.4 \mathrm{~mm}$ was evaluated as AP, and from 1.0 to $1.3 \mathrm{~mm}$ was determined as thickened intima. We also studied the blood flow velocity, as well as the nature, type, surface, length and location of the AP. All detected APs were evaluated as stable (calcified, homogeneous and heterogeneous) or unstable (with a rough surface, with ulceration and/or bleeding).

\section{Carotid artery specimens}

Group 2 patients with hemodynamically significant carotid stenosis and unstable APs underwent carotid endarterectomy (CEE) at the Tashkent Medical Academy. All samples were obtained immediately after CEE for determination of zinc level. Normal post-mortem carotid arteries (10 specimens) were used as control tissue.

\section{MMP-9 and TIMP-1 quantification}

In all patients, after CUS, blood was taken from the cubital vein in the morning on an empty stomach after a 12-hour overnight fast. All venous blood samples were immediately centrifuged and serums were frozen at $-20^{\circ} \mathrm{C}$. The serum concentration of MMP-9 and TIMP- 1 was determined using the standard test systems for immunoassay (BenderMedsystems GmbH, Austria) on the spectrophotometer Plate Reader (Hospitex Diagnostics, Italy).

\section{Quantification of zinc in serum, AP, and hair}

The serum Zn was determined colorimetrically by using a computerized, biochemical, automatic analyzer Mindray BS-200 (China) and kit «Zinc-Vital» (Vital Development Corporation, Russia). Quantitative determination of the zinc level in hair and AP was carried out by optical emission spectrometry with an inductively coupled argon plasma analyzer Optima 2100 DV (Perkin Elmer, USA).

\section{Serum lipid profile}

The total cholesterol (TC), low-density lipoprotein cholesterol (LDL-C) high-density lipoprotein cholesterol (HDL-C), and triglyceride (TG) levels were determined in the venous blood using automatic analyzer Mindray BS-200 (China).

Results were statistically processed. The mean (M) and standard error of the mean (SEM) were deduced. For data with normal distribution, inter-group comparisons were performed using Student's t-test. The Mann-Whitney (U Test) was used to compare the differences between the two independent groups (for nonparametric data). Pearson's correlation coefficient (r) was used to determine the strength of the relationship between the two continuous variables. A probability value of $P<0.05$ was considered statistically significant.

\section{Results}

Both groups were comparable in frequency of hypertension, hyperlipidemia, obesity, type 2 diabetes, coronary heart disease, previous MI, and age. In Group 1, chronic cerebral ischemia (CCI) stage I, II and IV was found in $35(77.8 \%), 1(2.2 \%)$, and $9(20 \%)$ patients, respectively. In Group 2, we found only the advanced CCI stages III and IV in 13(46.4\%) and 15(53.6\%) patients, respectively. In Group 1 , the number of men was 1.3 times less than in Group 2. In Croup 2, the incidence of stroke (57.1\%) was 2.6 times higher than in Group 1(22.2\%) (Table 1).

Differences in lipid profile were not significant (Table 2 ), probably because in Group 2 patients, it was 2.5 times more likely that statins were prescribed $(78.6 \%$ vs. $31.1 \%)$.

The level of $\mathrm{Zn}$ in serum and hair was not significantly different between Groups 1 and 2. However, $\mathrm{Zn}$ levels in unstable AP were lower than in the control group (Table 3).

The serum level of MMP-9 was 2.7 times higher in Group 2 compared to Group1 and the control group $(539.03 \pm 57.1 \mathrm{ng} / \mathrm{ml}$ vs. $200.9 \pm 8.5$ and $197.42 \pm 10.4 \mathrm{ng} / \mathrm{ml}$, respectively, $P<0.001$ ). 
Table 1.

Characteristics of the patient groups

\begin{tabular}{|l|c|c|}
\hline \multicolumn{1}{|c|}{ Variable } & Group 1 $(\mathrm{n}=45)$ & Group 2 $(\mathrm{n}=28)$ \\
\hline Age, years & $67.9 \pm 1.5$ & $62.8 \pm 1.2$ \\
\hline Male/Female & $30 / 15(67 \% / 33 \%)$ & $25 / 3(89 \% / 11 \%)^{*}$ \\
\hline Hypertension & $42(93.3 \%)$ & $26(92.9 \%)$ \\
\hline Hyperlipidemia & $22(48.9 \%)$ & $15(53.6 \%)$ \\
\hline Obesity & $19(42.2 \%)$ & $7(25 \%)$ \\
\hline Diabetes & $14(31.1 \%)$ & $14(50 \%)$ \\
\hline CHD & $36(80 \%)$ & $26(92 \%)$ \\
\hline History of MI & $6(13.3 \%)$ & $4(14.3 \%)$ \\
\hline History of stroke & $10(22.2 \%)$ & $16(57.1 \%)^{*}$ \\
\hline Body mass index & $27.7 \pm 0.59$ & $27.2 \pm 0.79$ \\
\hline IMT CCA, mm & $0.99 \pm 0.04$ & $1.2 \pm 0.05 *$ \\
\hline DCAS, $\%$ & $44.2 \pm 3.6$ & $75.4 \pm 2.9^{*}$ \\
\hline
\end{tabular}

${ }^{*} p<0.05$. IMT CCA - intima-media thickness of the common carotid artery, DCAS - degree of carotid artery stenosis.

Table 2.

Serum lipid profile of patients in the study groups

\begin{tabular}{|l|c|c|c|}
\hline \multicolumn{1}{|c|}{ Parameters } & Control group & Group 1 & Group 2 \\
\hline TC, $\mathrm{mmol} / 1$ & $5 \pm 0.5$ & $5.2 \pm 0.16$ & $4.6 \pm 0.18$ \\
\hline $\mathrm{TG}, \mathrm{mmol} / 1$ & $1.7 \pm 0.12$ & $1.8 \pm 0.12$ & $2.2 \pm 0.29$ \\
\hline $\mathrm{HDL}, \mathrm{mmol} / 1$ & $1.06 \pm 0.13$ & $0.94 \pm 0.02$ & $1.04 \pm 0.05$ \\
\hline LDL, $\mathrm{mmol} / 1$ & $3.8 \pm 0.4$ & $3.7 \pm 0.12$ & $3.2 \pm 0.15$ \\
\hline
\end{tabular}

Table 3.

The levels of $Z n$ in serum, scalp hair, and AP

\begin{tabular}{|l|c|c|c|}
\hline \multicolumn{1}{|c|}{ Biosubstrates } & Control group & Group 1 & Group 2 \\
\hline Serum, mmol/1 & $18.3 \pm 4.16$ & $13.7 \pm 1.4$ & $15.9 \pm 0.62$ \\
\hline Scalp hair, mg/g & $218.65 \pm 84.23$ & $220.5 \pm 17.07$ & $212.5 \pm 17.01$ \\
\hline AP, $\mathrm{mg} / \mathrm{g}$ & $148.55 \pm 15.3$ & - & $82.8 \pm 16.8^{*}$ \\
\hline
\end{tabular}

$* p<0.05$.

The serum level of TIMP-1 was twice as high in Group 2 compared to Group $1(2,410.2 \pm 123.8 \mathrm{ng} / \mathrm{ml}$ and $1,180.98 \pm 47.98 \mathrm{ng} / \mathrm{ml}$, respectively, $\mathrm{p}<0.001$ ). A significant difference was also found between Group 2 and the control group $(P<0.001)$. In Group 2, we found an increase in serum TIMP-1 level of about 2 times in comparison with the control group. However, we did not find statistically significant differences between Group 1 and the control group $(P>0.05)$.

The index of MMP-9/TIMP-1 was 1.6 times higher in Group $2(0.24 \pm 0.03)$ compared to the control group $(0.15 \pm 0.02)(P<0.02)$. Significant differences were not found between Group 1 and 2, or between Group 1 and the control group (Table 4).

Correlational analysis revealed a moderate positive correlation between DS with the serum levels of MMP-9 and TIMP-1 ( $\mathrm{r}=0.47$ and $\mathrm{r}=0.53$, respectively, $P<0.001$ ) (Fig.1,2). We also found a significant positive correlation between MMP-9 and TIMP-1(r=0.58, $P<0.001)$ (Fig.3).

\section{Table 4.}

Serum levels of MMP-9 and TIMP-1, and MMP-9/TIMP-1 ratio

\begin{tabular}{|c|c|c|c|c|c|c|}
\hline $\begin{array}{l}\text { Para- } \\
\text { meters }\end{array}$ & $\begin{array}{c}\text { Control } \\
\text { group }\end{array}$ & Group 1 & Group 2 & $\begin{array}{c}\mathrm{P} \\
(\mathrm{c}-1)\end{array}$ & $\begin{array}{c}\mathrm{P} \\
(\mathrm{c}-2)\end{array}$ & $\begin{array}{c}\mathrm{P} \\
(1-2)\end{array}$ \\
\hline $\begin{array}{c}\text { MMP-9 } \\
(\mathrm{ng} / \mathrm{ml})\end{array}$ & $197.42 \pm 10.4$ & $200.9 \pm 8.5$ & $539.03 \pm 57.1$ & $>0.05$ & $<0.001$ & $<0.001$ \\
\hline $\begin{array}{l}\text { TIMP-1 } \\
(\mathrm{ng} / \mathrm{ml})\end{array}$ & $1192.5 \pm 61.5$ & $1181.0 \pm 48.0$ & $2410.2 \pm 123.8$ & $>0.05$ & $<0.001$ & $<0.001$ \\
\hline $\begin{array}{l}\text { MMP-9/ } \\
\text { TIMP-1 }\end{array}$ & $0.15 \pm 0.02$ & $0.18 \pm 0.009$ & $0.24 \pm 0.03$ & $>0.05$ & $<0.02$ & $>0.05$ \\
\hline
\end{tabular}

$P(c-1)$ - between Group 1 and the control group; $P(c-2)$ - between Group 2 and the control group; P (1-2) - between Group 1 and Group 2.

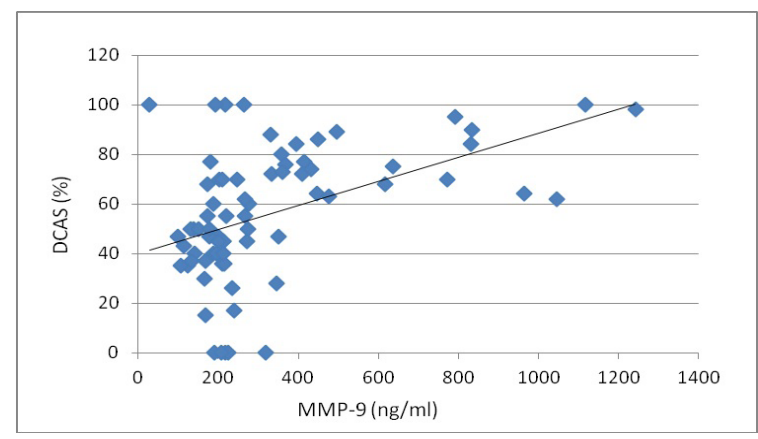

Figure 1. The correlation between MMP-9 and DCAS $(n=73, r=0.47 ; P<0.001)$

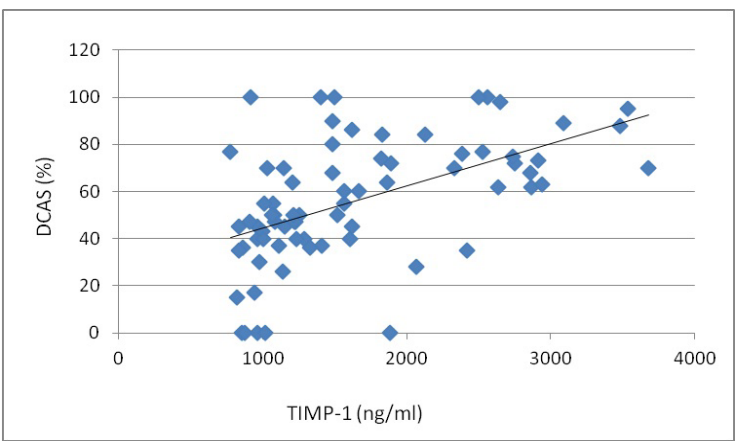

Figure 2. The correlation between TIMP-1 and DCAS $(n=73, r=0.53 ; P<0.001)$

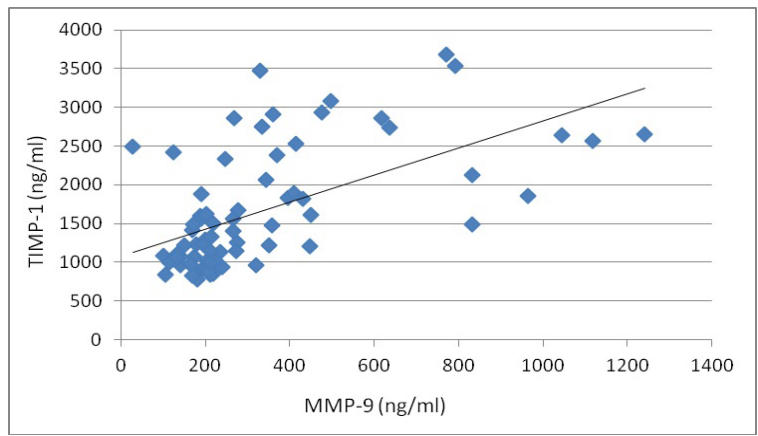

Figure 3. The correlation between MMP-9 and TIMP-1 $(n=73, r=0.58 ; p<0.001)$ 
Our results revealed no significant association of serum MMP-9 and TIMP-1 with age $(\mathrm{r}=-0.16$ and $\mathrm{r}=-0.11$, respectively, $P>0.05$ ).

We found a weak inverse correlation between the levels of serum zinc and its concentration in hair $(\mathrm{r}=-0.21$; $P=0.07$ ), a positive correlation between serum zinc and its concentration in the APs $(\mathrm{r}=0.21 ; P=0.07)$, a weak positive correlation between zinc in the hair and its level in the APs $(\mathrm{r}=0.23 ; P<0.05)$, and a negative correlation between serum zinc level and IMT CCA(r=-0.24; $P<0.05)$.

Concentration of zinc in bio-substrates also correlated with serum levels of MMP-9 and TIMP-1. We observed a weak negative correlation between serum MMP-9 and the levels of zinc in the APs $(\mathrm{r}=-0.25 ; P<0.05)$. The relationships between other parameters were not statistically significant.

\section{Discussion}

The results obtained show the increased serum concentrations of MMP-9 and TIMP-1 in patients with unstable AP. This result confirms the role MMP-9 and TIMP1 play in CAAP destabilization and as predictive markers of CAAP phenotype. Similar results were obtained by other authors [5-8, 13-14]. Furthermore, we found a significant positive correlation between serum levels of MMP-9/TIMP-1 and the degree of carotid stenosis, which is consistent with the findings of some other researchers [4, 14-17].

TIMP regulates strictly the activity of MMP-9[17]. We noted a significant positive correlation between serum MMP-9 and TIMP-1. The increased level of serum TIMP-1 in parallel with the serum level of MMP-9 can be explained as a compensatory response aimed at containing the activity of MMP-9 and the progression of atherosclerosis [18]. MMPs and TIMPs levels in early post-MI period may serve as estimates of post-MI cardiac damage and remodeling. MMP-9 and TIMP-1 correlate with echocardiographic parameters of left ventricular (LV) dysfunction after acute MI and may identify patients at risk of subsequent LV remodeling and adverse prognosis [19]. However, some researchers have not found an association between MMP-9 and TIMP-1 [16]. The index of MMP-9/TIMP-1 is used to evaluate the balance between MMP-9 and its inhibitor. According to Cheng et al. [20], in healthy individuals this ratio was $0.11 \pm 0.03$. In our study, this ratio was higher in all patients $(0.18 \pm 0.009$ in Group 1 and $0.24 \pm 0.03$ in Group 2) than in the control group $(0.15 \pm 0.02)$. In a patient with an embologenic fresh thrombus on the AP surface, this ratio increased even up to 0.47 (3 times higher than in the control group). These results indicate an imbalance towards MMP-9.

We found a significant negative correlation between IMT CCA and serum zinc, which corresponds to the results reported by other authors [21]. Existing data are contradictory regarding the negative or protective effects of zinc in the development of atherosclerosis [22-24]. In study of Stadler et al. [25], elevated levels of zinc (about 6-fold) were detected in advanced lesions compared to healthy tissue or early lesions. They found the highly-significant positive correlations between zinc and calcium in samples of carotid endarterectomy $(\mathrm{r}=0.895 ; P=0.0001)$. The reported protective effect of zinc accumulation is proposed to be associated with lesion calcification. It is known that highly fibrotic, calcified AP is less prone to rupture than lipid-rich, matrix-poor AP. In people with high levels of zinc, the decreased extent of cardiovascular complications may be due to calcium accumulation in a fibrous cap and hence the decreased propensity to AP ruptures.

Although there are many emerging biomarkers of inflammation and progression of atherosclerosis, their clinical utility remains unclear. Current evidence still favors the need for further investigation into the mechanisms through which these biomarkers may exert prognostic impact in patients with atherosclerotic lesions [26].

\section{Conclusions}

-High serum concentration of MMP-9 and TIMP-1, and MMP-9/TIMP-1 imbalance testify to CAAP instability.

-Increasing the serum concentration of MMP-9 is accompanied by decreasing the zinc level in AP.

-With the progression of atherosclerosis and increasing IMTCCA, the level of zinc in serum and APs decreases

-Reducing the concentration of zinc in the hair is accompanied by a decrease in zinc level in AP specimens.

\section{Acknowledgments}

I thank the surgeons of the Tashkent Medical Academy especially to Drs. Ravshan D. Sunnatov and Abdurasul A. Yulbarisov for the provision of the artery samples. I am grateful to Prof. Abdumalik N. Aripov and Prof. Gulnora A. Rozikhodjaeva for the advice.

\section{References}

1. Newby AC. Metalloproteinases and vulnerable atherosclerotic plaques. Trends Cardiovasc Med 2007; 17:253-8.

2. Găgalăte V, Ozheră̌tene V, Kalibatene D, Laurikenas $\mathrm{K}$, Sabaliauskene Z. Association between structure of atherosclerotic plaques in carotid arteries and myocardial infarction. Kardiologiia 2013; 53(9):21-5. [Article in Russian]. 3. Heo SH, Cho CH, Kim HO, Jo YH, Yoon KS, Lee JH, et al. Plaque rupture is a determinant of vascular events in carotid artery atherosclerotic disease: involvement of matrix metalloproteinases 2 and 9. J Clin Neurol 2011; 7(2):69-76.

4. Sapienza P, di Marzo L, Borrelli V, Sterpetti AV, Mingoli A, Cresti S, et al. Metalloproteinases and their inhibitors are markers of plaque instability. Surgery 2005;37(3):355-63.

5. Loftus IM, Naylor AR, Bell PR, Thompson MM. Plasma MMP-9 - a marker of carotid plaque instability. Eur J Vasc Endovasc Surg 2001;21(1):17-21.

6. Eldrup N, Grønholdt ML, Sillesen H, Nordestgaard BG. Elevated matrix metalloproteinase-9 associated with stroke or cardiovascular death in patients with carotid stenosis. Circulation 2006; 114(17):1847-54.

7. Zhou Z, Li X, Yang B, Jiang D. Relationship between lysophosphatidic acid and matrix metalloproteinase-9 plasma concentrations and carotid atheromatous plaque stability 
in patients with cerebral infarction. J Int Med Res 2014; 42(3):669-676.

8. Silvello D, Narvaes LB, Albuquerque LC, Forgiarini LF, Meurer L, Martinelli NC, et al. Serum levels and polymorphisms of matrix metalloproteinases (MMPs) in carotid artery atherosclerosis: higher MMP-9 levels are associated with plaque vulnerability. Biomarkers 2014; 19(1):49-55.

9. Zitka O, Kukacka J, Krizkova S, Huska D, Adam V, Masarik M, et al. Matrix metalloproteinases. Curr Med Chem 2010;17(31):3751-68.

10. Jacobsen FE1, Lewis JA, Cohen SM. The design of inhibitors for medicinally relevant metalloproteins. ChemMedChem 2007; 2(2):152-71.

11. Lukacova V, Zhang Y, Mackov M, Baricic P, Raha S, Calvo JA, et al. Similarity of binding sites of human matrix metalloproteinases. J Biol Chem 2004; 279(14):14194-200.

12. Eby GA, Halcomb WW. High-dose zinc to terminate angina pectoris: a review and hypothesis for action by ICAM inhibition. Med Hypotheses 2006; 66(1):169-72.

13. Tan C, Liu Y, Li W, Deng F, Liu X, Wang X, et al. Associations of matrix metalloproteinase- 9 and monocyte chemoattractant protein-1 concentrations with carotid atherosclerosis, based on measurements of plaque and intimaemedia thickness. Atherosclerosis 2014; 232(1):199-203. 14. Alvarez B, Ruiz C, Chacón P, Alvarez-Sabin J, Matas M. Serum values of metalloproteinase-2 and metalloproteinase- 9 as related to unstable plaque and inflammatory cells in patients with greater than $70 \%$ carotid artery stenosis. J Vasc Surg 2004; 40(3):469-75.

15. Beaudeux JL, Giral P, Bruckert E, Bernard M, Foglietti MJ, Chapman MJ. Serum matrix metalloproteinase-3 and tissue inhibitor of metalloproteinases- 1 as potential markers of carotid atherosclerosis in infraclinical hyperlipidemia. Atherosclerosis 2003; 169(1):139-46.

16. Gaubatz JW, Ballantyne CM, Wasserman BA, He M, Chambless LE, Boerwinkle E, et al. Association of circulating matrix metalloproteinases with carotid artery characteristics: the Atherosclerosis Risk in Communities Carotid MRI Study. Arterioscler Thromb Vasc Biol 2010; 30(5):1034-42.
17. Romero JR, Vasan RS, Beiser AS, Polak JF, Benjamin EJ, Wolf PA, et al. Association of carotid artery atherosclerosis with circulating biomarkers of extracellular matrix remodeling: the Framingham Offspring Study. J Stroke Cerebrovasc Dis 2008; 17(6):412-417.

18. Golovkin AS, Matveeva VG, Grigor'ev EV, Baĭrakova $\mathrm{IuV}$, Shukevich DL, Velikanova EA, et al. Postoperative dynamic changes in matrix metalloproteinase levels in patients with coronary artery bypass graft procedure complications. Kardiologiia 2012; 52(9):4-7. [Article in Russian]

19. Kelly D, Khan SQ, Thompson M, Cockerill G, Ng LL, Samani N, et al. Plasma tissue inhibitor of metalloproteinase-1 and matrix metalloproteinase-9: novel indicators of left ventricular remodelling and prognosis after acute myocardial infarction. Eur Heart J 2008; 29(17):2116-24.

20. Cheng M, Hashmi S, Mao X, Zeng QT. Relationships of adiponectin and matrix metalloproteinase-9 to tissue inhibitor of metalloproteinase-1 ratio with coronary plaque morphology in patients with acute coronary syndrome. Can J Cardiol 2008; 24(5):385-90.

21. Ari E, Kaya Y, Demir $\mathrm{H}$, et al. The correlation of serum trace elements and heavy metals with carotid artery atherosclerosis in maintenance hemodialysis patients. Biol Trace Elem Res 2011; 144(1-3):351-9.

22. Beattie JH, Kwun IS. Is zinc deficiency a risk factor for atherosclerosis? Br J Nutr 2004; 91(2):177-81.

23. Hughes S, Samman S. The effect of zinc supplementation in humans on plasma lipids, antioxidant status and thrombogenesis. J Am Coll Nutr 2006; 25(4):285-91.

24. Vasto S, Mocchegiani E, Candore G, Listì F, ColonnaRomano G, Lio D, et al. Inflammation, genes and zinc in ageing and age-related diseases. Biogerontology 2006; 7(56):315-27.

25. Stadler N, Stanley N, Heeneman S, et al. Accumulation of zinc in human atherosclerotic lesions correlates with calcium levels but does not protect against protein oxidation. Arterioscler Thromb Vasc Biol 2008; 28(5):1024-30.

26. Doo Sun Sim, Youngkeun Ahn. Novel inflammatory biomarkers in acute coronary syndrome. Korean J Intern Med 2013; 28(2):156-8. 